\title{
Analysis of Multi-Signal Hyperspectral Datasets Collected by EPMA
}

\author{
N. C. Wilson ${ }^{1}$, C. M. MacRae ${ }^{1}$ and A. Torpy ${ }^{1}$ \\ 1. CSIRO Mineral Resources, Microbeam Laboratory, Private Bag 10, Clayton South, Victoria, Australia
}

Since the first electron probe microanalyser was developed by Castaing[1] there have been numerous developments to increase its capabilities in performance and data collection. Mapping has become an important use of the EPMA, and in recent years developments in detectors and computing technology has allowed the collection of hyperspectral maps. The latest hyperspectral detector to become available is the soft x-ray emission spectrometer (SXES) [2]. This detector collects a limited range of the X-ray spectrum in parallel, but has lowered the minimum energy of detectable x-rays to as low as $\mathrm{Mg}-\mathrm{L}$ (49 $\mathrm{eV}$ ), with an energy resolution as low as $99 \mathrm{meV}$ for $\mathrm{Mg}-\mathrm{L}$. This not only opens up the opportunity for direct lithium measurement in the microprobe, but also allows for the spectral mapping of L, M, and N lines of some elements. These low energy lines originate from transitions of electrons from orbitals that are involved in bonding, and thus the peak shape and position of these lines are sensitive to the local chemical environment.

We have fitted a field-emission gun EPMA (JEOL 8530F) with a SXES detector, two SDD EDS detectors (Bruker XFLASH 6010) and a high resolution grating CL system [3]. In the EPMA none of these detectors obscure one another, so we have developed software that allows the simultaneous collection of the backscatter electron signal, WDS, EDS, SXES and CL spectral data. This gives us a hyperspectral data set that contains information from a large part of the emitted energy spectrum. The simultaneous acquisition of all signals has the advantage of avoiding pixel misalignment in maps, and minimises the electron beam induced damage artefacts associated with multiple pass mapping. In addition to detector improvements, the EPMA column continues to improve. To improve the ability to perform high resolution mapping, field-emission gun columns are advancing, with increasing amounts of current available at low accelerating voltages, while still maintaining high spatial resolution. Other recent advances in the EPMA column is the ability to set a controlled sub-micron defocus, giving the ability to choose a spot size between fully focused and one micron. This use of this is advantageous in reducing beam damage during microanalysis on fine features or during high resolution mapping.

The collection of hyperspectral maps allows software tools to be used for the post-hoc examination of the data set. This can lead to the discovery of unexpected phases (in x-ray data), bonding changes (in the SXES data) and defects or centers (in the cathodoluminescence data) within a sample. We use data clustering algorithms [4] to analyse hyperspectral data, and an example of this is given in Fig 1. The map is of a weathered sulphide mineral sample from Kabwe, Zambia, containing sphalerite ( $\mathrm{ZnS})$ and pyrite $\left(\mathrm{FeS}_{2}\right)$ and was collected at $7 \mathrm{kV}$, with a beam current of $150 \mathrm{nA}$ a dwell time of $800 \mathrm{~ms}$ and a step size of 2 micron. The SXES used a 200N grating which spanned an energy range of 64-350eV, and the CL spectrometer had a grating sensitive from $200-950 \mathrm{~nm}$. Fig. $1 \mathrm{~b}$ shows the phase summed spectra for the three sulphides identified. Summing all the pixels in a phase has the advantage of giving low noise spectra that take time to acquire, but without the damage artefacts that single point analysis would induce. The CL spectra shows, for example, in the sphalerite a peak at $2.2 \mathrm{eV}$, associated with $\mathrm{Mn}^{2+}$ and a minor peak at $1.35 \mathrm{eV}$ reflecting the bandgap. A region of the SXES spectrum around the S Ll line is shown in Fig 1d, displaying the peak shifting and shape change due to bonding. A further advantage of hyperspectral data collection is that if the WDS peaks are used for quantification, the background can be 
extracted from the phase summed EDS spectra to give a low noise alternative to using the mean atomic number background correction method.

(a)

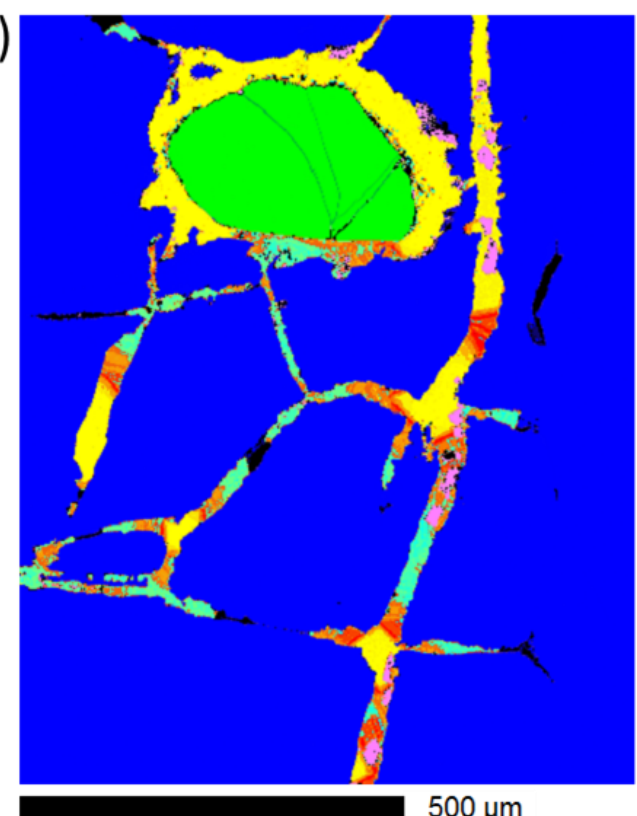

(d)

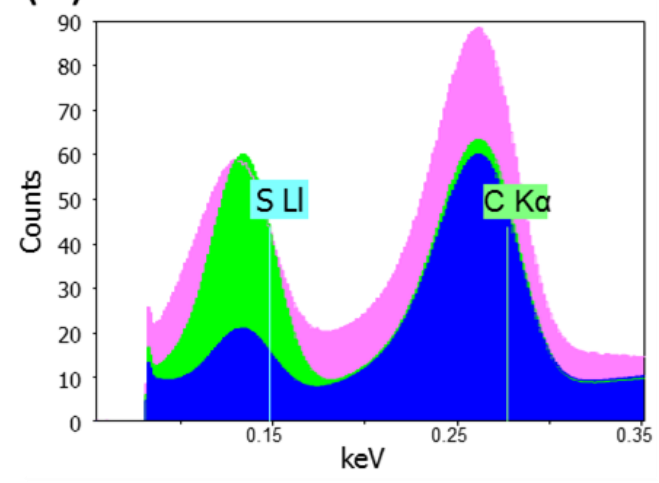

(b)

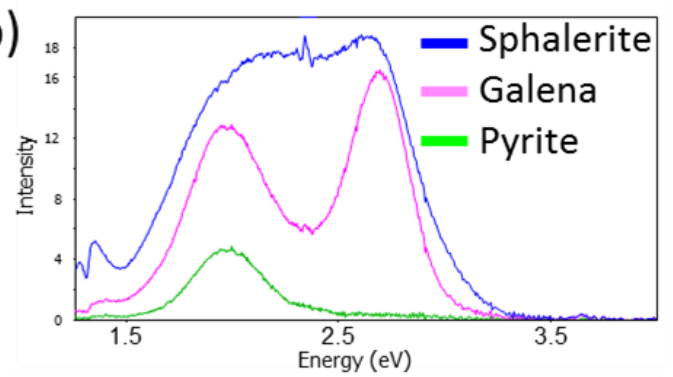

Sphalerite

Pyrite

$\mathrm{Zn}$ carbonate

$\mathrm{Zn}$ carbonate $(\mathrm{Fe})$

$\mathrm{Zn}$ carbonate $(\mathrm{Mg}, \mathrm{Fe})$

Galena

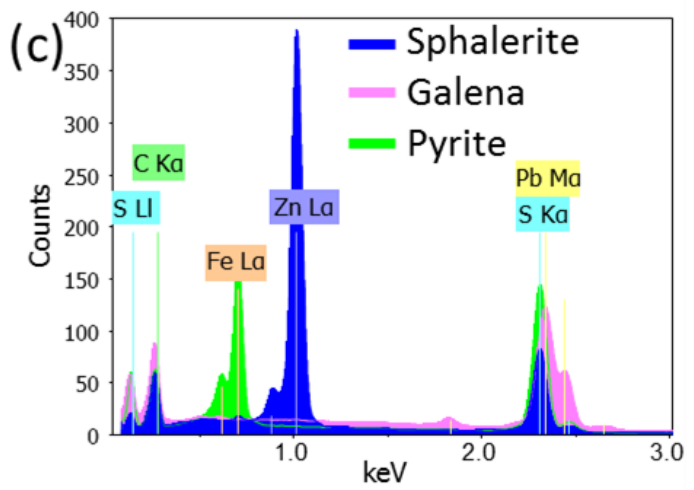

(e)

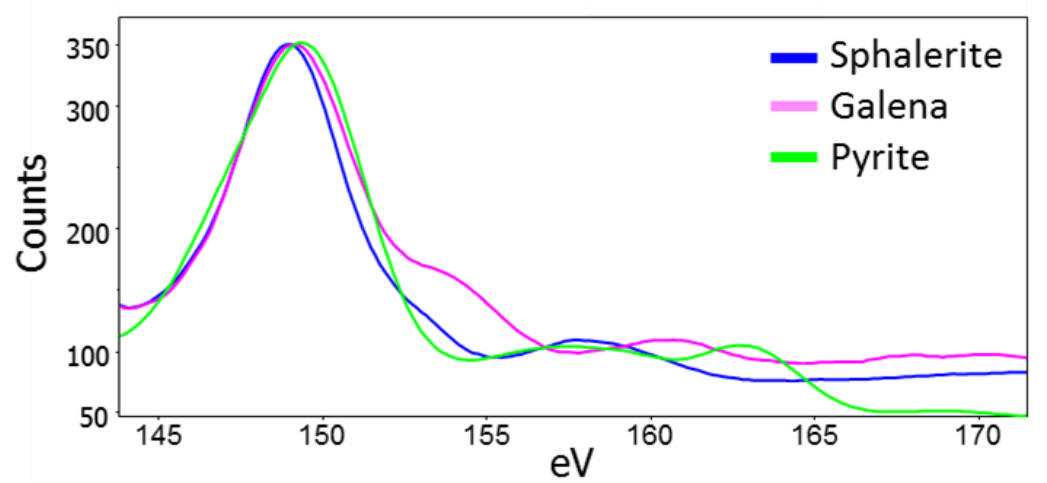

Figure 1. (a) Chemical phase-patched image of a sulphide mineral sample. (b) CL spectra of the sulphide phases extracted from the cluster data. (c) EDS spectra from the sulphide phases and (d) a zoomed section of the EDS around S Ll and C Ka. (e) SXES spectra from the sulphide phases

References:

[1] R. Castaing, PhD Thesis University of Paris.

[2] M. Terauchi et al., Journal of Electron Microscopy 61 (1) (2012) p. 1-8.

[3] We acknowledge support from ARC - LE130100087.

[4] N. C. Wilson et al, Microscopy and Microanalysis 14 (Suppl. 2) (2008) p. 764. 